\title{
Application of Perfect Difference Codes in Wireless Infrared Systems
}

\author{
Fahim A. Umrani, Salah Obayya \\ Faculty of Advanced Technology, University of Glamorgan, Pontypridd, UK \\ E-mail: \{faumrani, sobayya\}@glam.ac.uk \\ Received February 8, 2010; revised May 3, 2010; accepted June 19, 2010
}

\begin{abstract}
In this paper, we apply the perfect difference codes in wireless infrared systems considering the diffuse indoor optical wireless configuration. The bit error rate performance of the uplink wireless infrared system using Gaussian approximations is analyzed taking into account the effects of multiple-access interference, the ambient light noise, and the dark current. The proposed system also uses the compact encoder and decoder architecture resulting in a low cost system.
\end{abstract}

Keywords: OCDMA, Compact Encoder/Decoder, Perfect Difference Codes

\section{Introduction}

The past few decades have seen the increased interest of the researchers in the utilization of infrared (IR) frequencies for short range wireless communications [1-5]. Wireless radio technologies have been designed and implemented comparatively over a much longer time than wireless infrared systems, however, the low complexity and low cost of infrared systems make relatively new IR systems very attractive and cost effective solution at a bit rates up to several tens of Mbps. Many potential applications for this technology, such as Wireless LANs, have already been suggested. Optical wireless networks are suitable for "campus" networking, metropolitan communication infrastructure, rapid deployment in emergency situations, disaster recovery, and in military contexts.

The diffuse indoor optical wireless configuration (i.e. non-directed, non-line of sight) is one of the most convenient and robust solution for local area networks (LAN). In the diffuse configuration, the transmitters and the receivers of infrared systems do not require to be carefully aligned, nor do they need to be in a line-of-sight (LOS) path so that communication can be maintained. The other major advantages of diffuse systems are their flexibility and the roaming they allow in a room. This flexibility makes them the ideal choice for ad hoc networks and gives the end users freedom to roam freely insider the office or room. Therefore, in this paper, we consider a diffuse configuration. However, this freedom of roaming results in the problems associated with high path loss and inter-symbol interference (ISI) due to multipath disper- sion. In a code-division based WIR systems, the effects of ISI can be compensated by using efficient spreading sequences, such as perfect difference codes (PDC) in spectral-amplitude coding based system [6].

Perfect difference codes are the special type of cyclic difference set with weight (number of pulsed chips) equals to $w$, length $v=w^{2}-w+1$, and correlation constraint of $\gamma=1$. The detailed information about the perfect difference codes can be found in [7-9]. PDCs provide a compromise performance between the two typical classes of unipolar sequences used in OCDMA systems, i.e., Optical Orthogonal codes and Prime codes, in terms of cardinality, sequence length and cross correlation constraint. PDCs have cross correlation constraint equal to OOC and sequence length comparable to Prime codes with the added advantage that PDCs can be constructed for any number of weight, while in Prime codes the weight is equal to a prime number. To the author's best knowledge most work on WIR systems adopting unipolar sequences is based on either OOC and Prime codes [10-11], we for the first time in this paper apply PDCs in wireless infrared systems. For the purpose of this paper we are interested in the following two properties of PDCs: 1) The cross correlation between the two PDCs is unity. This property is exploited to design the decoder to efficiently recover data by suppressing MAI effect, and 2) Perfect difference codes are cyclic shifted. The cyclic nature of PDCs is combined with the cyclic nature of Arrayed-waveguide multiplexers to construct compact efficient encoders.

In this paper, we apply perfect difference codes for the 
first time in indoor optical wireless channel. A wireless infrared system is proposed which uses the compact encoders and decoders. The uplink performance analysis of such system is analyzed in terms of bit error rate versus path loss and bit error rate versus number of users.

Following this, in Section 2 we give the reader an introduction and overview of the system model explaining the channel model and transmitter receiver structure to clarify the analysis presented in the next section. We give the schematic diagram of the encoding and decoding device and explain how they work. In Section 3, we obtain the system performance under Gaussian approximations using the proposed model in Section 2. Finally Section 4 concludes the paper.

\section{System Model}

The number of active users is $I+1$ and $N_{\max }$ is maximum allowed number of users which can be associated to each station. We use PDC based OCDMA as uplink multiple access technique. The average received power using PDC is expressed by [12]

$$
P_{r}=\frac{w}{2 v} I_{r} A_{d}
$$

where $\quad I_{r}=$ is the received light intensity,

$A_{d}=$ is the area of photodetector.

\subsection{Channel Model}

One of the most important parameters that affect the performance of infrared system is the channel path loss which is the DC-gain $\left(H_{0}\right)$ of the channel transfer function. It can be expressed as:

$$
P_{r}=H_{0} P_{t}
$$

relating the transmitted and received average powers.

Figure 1 illustrates the non-directed non-line of sight configuration selected for the system under study in which the transmitter and receiver are pointed straight upward and transmitter emits a Lambertian pattern. The $h_{1}$ and $h_{2}$ represents the distance of transmitter and receiver from the ceiling, with diffuse reflectivity $\rho$, respectively. We assume that the diffuse reflectivity of ceiling is $80 \%$. The path loss for diffuse link is plotted in Figure 2 which is measured in a typical office [4].

\subsection{Transmitter \& Receiver}

At the transmitter on-off keying intensity modulation scheme is adopted and direct detection at the receiver. Duration of each chip is $T_{c}=T_{b} / v$ where $v$ is the code length and $T_{b}$ is the bit duration.

Figure 3 shows the proposed encoder designed with PDCs for $v=7$ and $w=3$. It contains $1 \times 7$ splitter and one $7 \times 7$ AWG router which can generate seven codewords simultaneously. After the light is incident on the splitter it is directed to 3 specific AWG input ports according to the adopted PDC. By controlling the states of $2 \times 2$ switches denoted as $d_{k}$ with the user's information bits, the encoder can transmit suitable codewords to the end users. The upper arm of $2 \times 2$ switch is connected to the combiner for broadcast transmission, while the lower arm is left unused. When the data bit is 1 a unique PDC assigned to each user is sent, however, when data bit is 0 , a common zero code assigned to all users is transmitted [13].

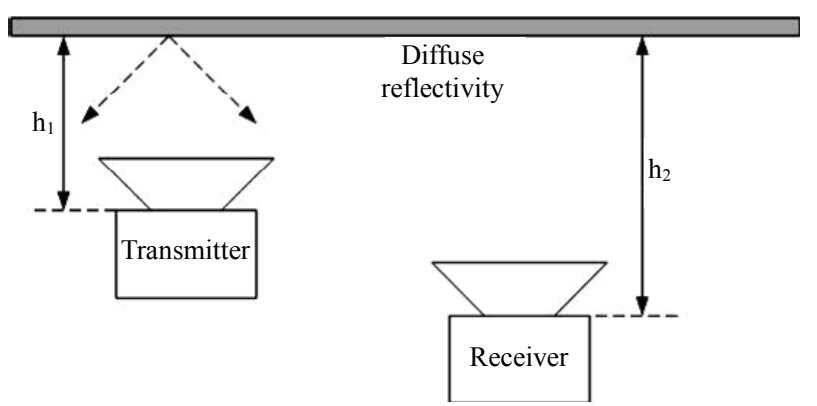

Figure 1. Non-directed non-line of sight LOS (diffuse) configuration.

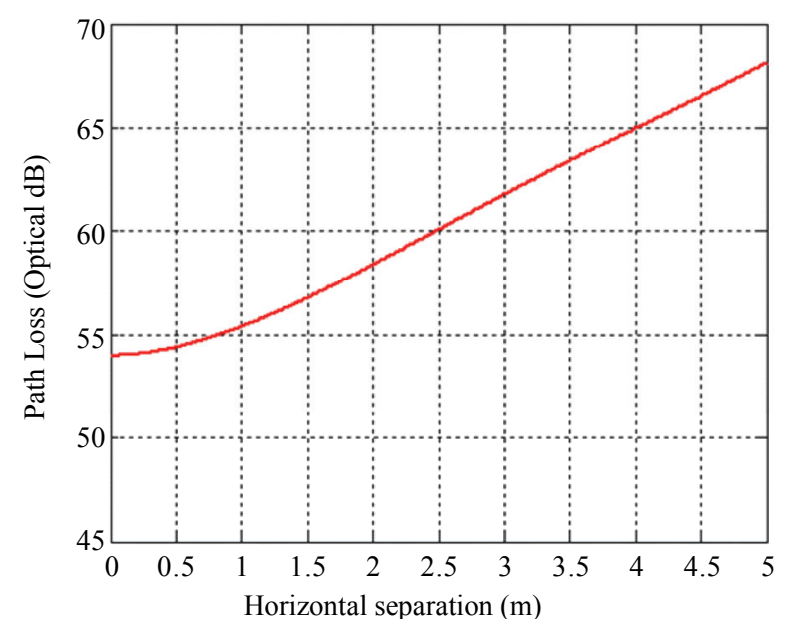

Figure 2. Optical path loss of a diffuse infrared links employing a Lambertian transmitter and a detector of area $A_{d}=$ $1 \mathrm{~cm}^{2}$ and reflectivity of $80 \%$ measured in a typical room.

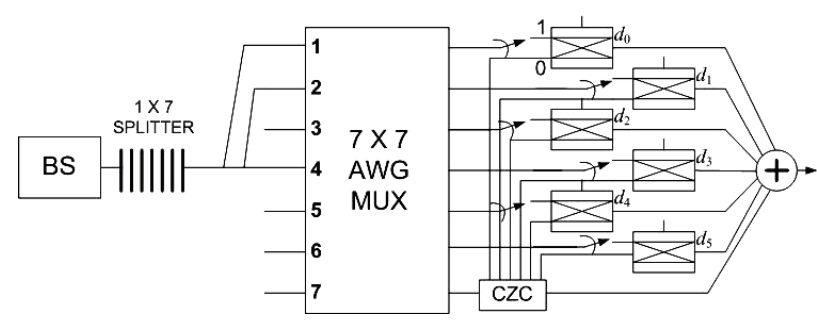

Figure 3. Encoder based on PDC. (BS: Broadband source). 
At the receiver, a composite signal containing the desired signal along with the noise and interference from all other $I$ active users passed through the channel is detected. The receiver's structure as illustrated in Figure $\mathbf{4}$ is based on correlation detection. The received signal is divided into $\mathrm{v}$ branches and then delayed to accumulate the marked chips in the optical correlator. At the receiver all the weighted chips of the desired sequence are summed to form a decision variable. This decision variable is compared to a threshold to detect data bit 1 or 0 [14].

\section{Performance Analysis and Results}

In this analysis, it is assumed that different nodes transmit asynchronously and independently. Further to make things simpler, different signals are assumed to be chip synchronous, which is a pessimistic case and gives an upper bound to the BER of the real asynchronous system $[14,15]$.

The number of interfering pulses received in $j_{t h}$ pulse position of the desired codeword is denoted by $\phi_{j}=1,2$, $\ldots, K$. The vector of received interference is denoted by $\vec{\phi}=\left(\phi_{1}, \phi_{2}, \ldots, \phi_{K}\right)$. In light of the cross-correlation property of PDC, two code-words cannot overlap at more than one pulse position. Therefore, the probability that two codewords overlap at one pulse position is $q=$ $w^{2} / 2 v$ where factor $1 / 2$ accounts for the probability that interfering user sent "one" only half time.

Given $I$ interfering users, the BER of the desired user's signal can be expressed as $[15,16]$ :

$$
P_{e}=\sum_{i=0}^{I} P_{r}(i) P\left(F_{i}\right)
$$

where $P_{r}(i)$ is the probability that there are $l$ interfering pulses, which is given by:

$$
P_{r}(i)=\left(\begin{array}{l}
I \\
i
\end{array}\right) q^{i(1-q)^{I-i}}
$$

$F_{i}$ is the set of all possible $\vec{\phi}$ vectors. Since the $I$ interfering users are not distinguishable in correlation receiver, $P\left(F_{i}\right)=P(\vec{\phi})$.

Using Gaussian approximation for photon detection, $P(\vec{\phi})$ can be written as $[15,16]$ :

$$
P(\vec{\phi})=0.5 Q\left(\frac{\theta-\mu_{0}}{\sqrt{\sigma_{0}}}\right)+0.5 Q\left(\frac{\mu_{1}-\theta}{\sqrt{\sigma_{1}}}\right)
$$

where

$$
Q(x)=\frac{1}{\sqrt{2 \pi}} \int_{x}^{\infty} e^{-\frac{y^{2}}{2}} d y
$$

$\mu_{b}$ and $\sigma_{b}$ is the mean and variance, respectively, of the signal when data bit $b(0$ or 1$)$ is transmitted, and $\theta$ is the threshold.

Let $P_{r}$ be the received optical power per chip at the input of correlator, then power at the output of optical correlator can be written as:

$$
P_{C R}= \begin{cases}P_{r}(w+I), & \text { for } b=1 \\ P_{r}(I), & \text { for } b=0\end{cases}
$$

here $w$ is the weight of the PDC, $I$ is the number of interfering users in the system.

The threshold $P_{t h}$ of the hard limiter is set to $P_{r}(w+$ $2 I) / 2$; therefore, the power $P_{r}(w+I)$ in Equation (7) is clipped to $P_{r}(w+2 I) / 2$ while the power $P_{r}(I)$ is clipped to zero. After that, the output of the hard limiter is transformed to an electronic signal by the photodetector. Finally, the integrate-and-dump circuit and the threshold circuit decide whether the received bit is 0 or 1 .

The average photon arrival rate $\lambda$ per pulse at the input of the optical correlator is given by $\lambda=\eta P_{r} / h f$. According to correlation property of PDC each user contributes one spectral component in the desired user's signal and the mean photon count produced by the $i_{\text {th }}$ interferer, which is a function of $\rho_{i}$ (path loss) and transmission power, is given is:

$$
I(\rho)=\sum_{k=1}^{K} i_{k}, \quad(k=1,2, \ldots N-1)
$$

Given $N=I$ and the desired bit $b=1$, using Gaussian expression, the mean and variance can be expressed as:

$$
\begin{aligned}
& \mu_{1}=G T_{c}\left(\frac{(k+2 I) \lambda}{R_{b}}+\frac{k I_{d}}{e}\right)+G T_{c}\left(\frac{\eta A_{d} m_{b}}{h f}\right) \\
& \sigma_{1}^{2}=G^{2} F_{e} T_{c}\left[\frac{(k+2 I) \lambda}{R_{b}}+\frac{k I_{d}}{e}\right]+G T_{c}\left(\frac{\eta A_{d} m_{b}}{h f}\right)+\sigma_{t h}^{2}
\end{aligned}
$$

where $A_{d}$ is the photodetector's area, $m_{b}$ is the mean photon count of the ambient light noise, and $I_{d}$ is the dark current, $G$ is the average APD gain of upper APD. $T_{c}$ is the chip duration, $I_{b}$ is the bulk leakage current, $I_{s}$ is the surface leakage current, $e$ is the electron charge, $F_{e}$ is the excess noise factor given as:

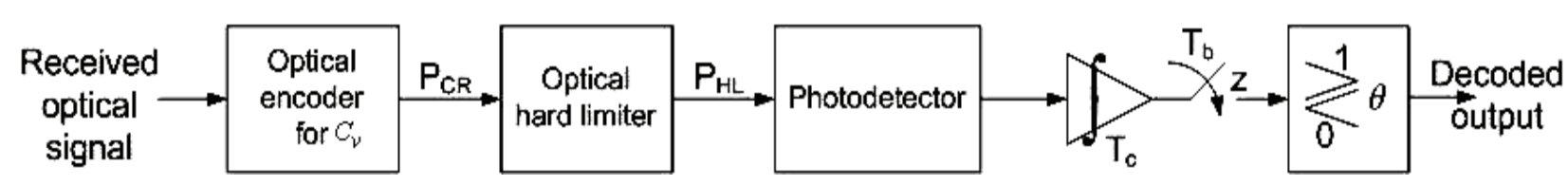

Figure 4. Decoder of the system. 


$$
F_{e}=k_{e f f} G+(2-1 / G)\left(1-k_{e f f}\right)
$$

here $k_{\text {eff }}$ is the APD effective ionization ratio and, $\sigma_{t h}^{2}$ is the variance of thermal noise given as:

$$
\sigma_{t h}^{2}=\frac{2 K_{b} T_{n} T_{c}}{e^{2} R_{L}}
$$

where $K_{b}$ is the Boltzman's constant, $T_{n}$ is the receiver noise temperature, and $R_{L}$ is the receiver load resistance.

Given $N=I$ and the desired bit $b=0$, using Gaussian expression, the mean and variance can be expressed as:

$$
\begin{aligned}
& \mu_{0}=G T_{c}\left(\frac{k I_{d}}{e}\right)+G T_{c}\left(\frac{\eta A_{d} m_{b}}{h f}\right) \\
& \sigma_{0}^{2}=G T_{c}\left[\frac{k I_{d}}{e}\right]+G T_{c}\left(\frac{\eta A_{d} m_{b}}{h f}\right)+\sigma_{t h}^{2}
\end{aligned}
$$

To minimize the BER, the optimal threshold $\theta$ can be derived as:

$$
\theta= \begin{cases}\left(\mu_{1}+\mu_{0}\right) / 2, & \text { for } \sigma_{1}^{2}=\sigma_{0}^{2} \\ \frac{b+\sqrt{b^{2}-a c}}{a}, & \text { for } \sigma_{1}^{2} \neq \sigma_{0}^{2}\end{cases}
$$

where

$$
\begin{gathered}
a=\sigma_{1}^{2}-\sigma_{0}^{2} \\
b=\sigma_{1}^{2} \mu_{0}-\sigma_{0}^{2} \mu_{1} \\
c=-\ln \left(\frac{\sigma_{1}^{2}}{\sigma_{0}^{2}}\right) \sigma_{0}^{2} \sigma_{1}^{2}-\sigma_{0}^{2} \mu_{1}^{2}+\sigma_{1}^{2} \mu_{0}^{2}
\end{gathered}
$$

Now, we present some numerical results for the wireless infrared system discussed above. We assume an infrared CDMA system with data rate of $R_{b}=2 \mathrm{Mbps}$ per user and PDC codewords with length $v=183$ and weight $w=14$. The system is operating at the wavelength of $\lambda=850 \mathrm{~nm}$, the ambient light noise intensity is $m_{b}=490 \mu \mathrm{W} / \mathrm{cm}^{2}$ the quantum efficiency of the Photodetector is $\eta=0.6$, Photodetector's area is $A_{d}=1$ $\mathrm{cm}^{2}$ and dark current is assumed to be $I_{d}=160 \mathrm{nA}$. The other parameters are listed in Table 1.

Figure 5 illustrates the bit error rate performance of the indoor optical distance versus the user's distance $(\rho)$ from the base station for transmission powers of $5 \mathrm{~mW}$, $20 \mathrm{~mW}, 15 \mathrm{~mW}$ and $30 \mathrm{~mW}$. The number of interfering users is kept fixed. One can see the near-far problem in a basic PDC wireless infrared network without power control. As the distance from the base station is increased the system performance degrades rapidly. This shows that the users which are near the base station obtain much more BER than needed but the data from far users can not be detected with desired BER. This generates the requirement of a power control algorithm in indoor wireless system to make sure that all users regardless of their distance from the base station can access the base station with equal power.

Figure 6 plots the error probability versus user's distance from the base station for different bit rates $(2,4$ and $10 \mathrm{Mbps}$ ). The transmitted power and the number of interferes is kept fixed at $P_{t}=20 \mathrm{~mW}$ and 5, respectively.

Table 1. Parameters used.

\begin{tabular}{ll}
\hline PD quantum efficiency & $\eta=0.6$ \\
Gain of the APD & 100 \\
Receiver noise temperature & $T n=300 \mathrm{~K}$ \\
Receiver load resistor & $R L=1030 \Omega$ \\
\hline
\end{tabular}

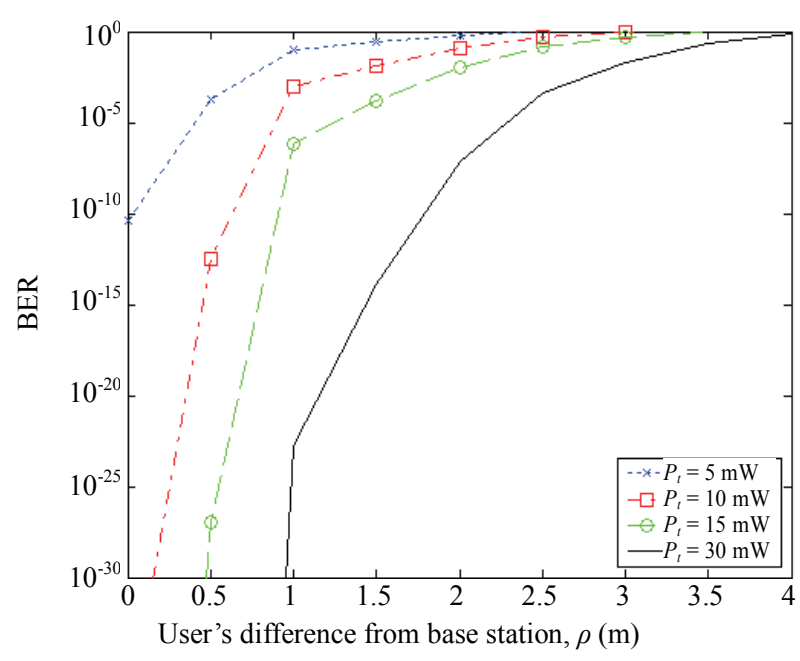

Figure 5. BER versus for various transmission powers. $\mathrm{Nu}$ mber of interferes is 5 .

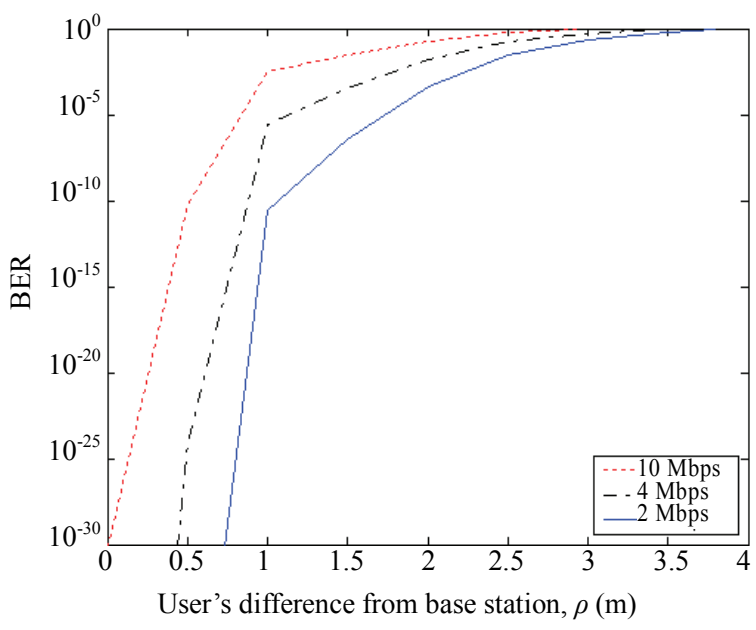

Figure 6. BER versus user's distance from base station $(\rho)$ for various bit rates. Number of interferes is 5 and $P_{t}$ $=20 \mathrm{~mW}$. 


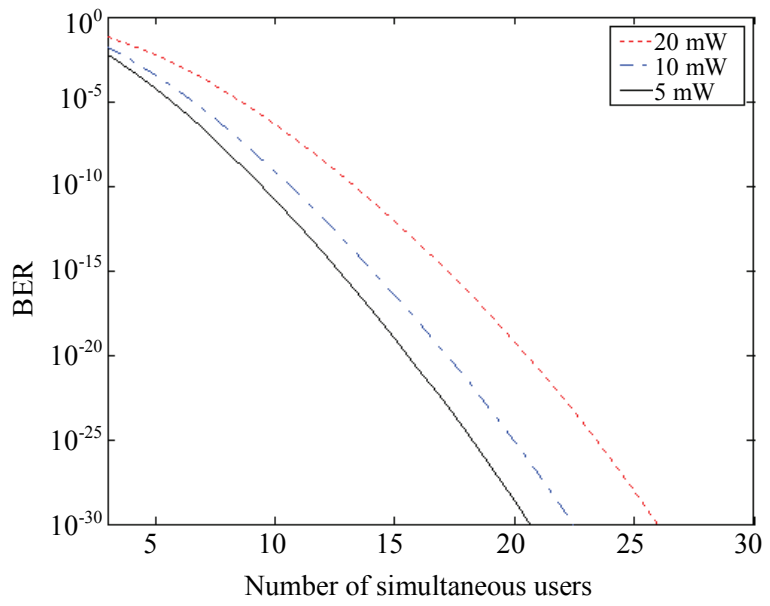

Figure 7. Error probability versus number of users for data rate of 2 Mbps.

It can be seen that the path loss as the user's move away from the base station puts severe restrictions on the data rate. In Figure 7, the bit error rate versus number of users is plotted. We can see that as the number of users increase the bit error rate performance is improved. The reason for this again can be found in Equation (7) which shows the increase in the number of users is directly proportional to the difference between data bit 1 and 0 .

\section{Summary}

In this paper, we applied perfect difference codes in wireless domain. The uplink performance in presence of various noise sources such as path loss, ambient noise, multiple access interference and thermal noise is analyzed. The bit error rate performance was analyzed over different transmission powers and different data rates. It is revealed from the results that an effective power control algorithm is required to mitigate path loss effects.

\section{Acknowledgements}

This research is supported by Mehran University of Engineering \& Technology, Pakistan.

\section{References}

[1] F. R. Gfeller, and U. Bapst, "Wireless in-House Data Communication via Diffuse Infrared Radiation," Proceedings of the IEEE, Vol. 67, No. 11, 1979, pp. 1474-1486.

[2] J. R. Barry, J. M. Kahn, et al., "Simulation of Multipath Impulse Response for Indoor Wireless Optical Channels," IEEE Journal on Selected Areas in Communications, Vol. 11, No. 3, 1993, pp. 367-379.
[3] J. B. Carruthers and J. M. Kahn, "Modelling of Nondirected Wireless Infrared Channels," IEEE Transactions on Communications, Vol. 45, No. 10, 1997, pp. 1260-1268.

[4] J. M. Kahn, P. Djahani, A. G. Weisbin, K. T. Beh, A. P. Tang and R. You, "Imaging Diversity Receivers for High-Speed Infrared Wireless Communications," IEEE Communications Magazine, Vol. 36, No. 12, 1998, pp. 88-94.

[5] Z. Ghassemlooy and A. C. Boucouvalas, "Guest Editorial: Indoor Optical Wireless Communications System and Networks," International Journal of Communication Systems, Vol. 18, No. 3, 2005, pp. 191-193.

[6] F. A. Umrani and S. Obayya, "New Encoder/Decoder Design for Spectral Amplitude Coding Based Optical Code Division Multiplexing (OCDM) System with Common Zero Code," submitted for publication.

[7] X.-S. Weng and J. S. Wu, "Perfect Difference Codes for Synchronous Fiber-Optic CDMA Communication Systems," Journal of Lightwave Technology, Vol. 19, No. 2, 2001, pp. 186-194.

[8] M. Hall, Jr., "Combinatorial Theory," Blaisdell Publishing Company, London, 1967.

[9] H. J. Ryser, "Combinatorial Mathematics," Wiley, New York, 1963.

[10] T. Satoh and T. Hashimoto, "Performance Evaluation of Chip-Synchronous, Code-Asynchronous Optical CDMA Systems for Indoor Infrared Wireless Communications with Diffuse Link," Electronics and Communications in Japan, Vol. 86, No. 4, 2002, pp. 75-88.

[11] B. M. Ghaffari, M. D. Matinfar and J. A. Salehi, "Wireless Optical CDMA LAN: Digital Implementation Analysis," IEEE Journal on Selected Areas in Communications, Vol. 27, No. 9, 2009, pp. 1676-1686.

[12] S. Zahedi, J. A. Salehi and M. Nasiri-Kenari, "A Photon Counting Approach to the Performance Analysis of Indoors Wireless Infrared CDMA Networks," Proceedings of IEEE Personal, Indoor and Mobile Radio Communications, London, Vol. 2, 18-21 September 2000, pp. 928932.

[13] L.-L. Jau and Y.-H. Lee, "Optical Code-Division Multiplexing Systems Using Common-Zero Codes," Microwave and Optical Technology Letters, Vol. 39, No. 2, 2003, pp. 165-167.

[14] J. A. Salehi and C. A. Brackett, "Code Division Multiple Access Techniques in Optical Fiber Networks-Part II: Systems Performance Analysis," IEEE Transactions on Communications, Vol. 37, No. 8, 1989, pp. 834-842.

[15] A. Aminzadeh-Gohari and M. R. Pakravan, "Analysis of Power Control for Indoor Wireless Infrared CDMA Communication," Proceedings of 25th IEEE International Performance, Computing, and Communications Conference, Phoenix, Vol. 6, 10-12 April 2006, pp. 297-302.

[16] M. Azizoglu, J. A. Salehi and Y. Li, "Optical CDMA via Temporal Codes," IEEE Transactions on Communications, Vol. 40, No. 7, 1992, pp. 1162-1170. 